\section{Disappearance of a large pedunculated polyp after diagnostic colonoscopy}

A 66-year-old Korean man was referred for colonic polypectomy. A colonoscopy performed at another hospital 1 week previously had revealed a large pedunculated polyp with an erythematous surface that was occupying almost the entire lumen in the mid-sigmoid colon ( Fig. 1). The polyp appeared hyperplastic, and no biopsy was performed.

The patient was not taking any nonsteroidal anti-inflammatory drugs, steroids, antibiotics, or other medication. No significant abnormality was found in his past history, family history, or physical examination. All laboratory results, including a complete blood cell count, blood biochemistry, and carcinoembryonic antigen (CEA), were within their normal ranges.

The patient underwent a second colonoscopy so that a polypectomy could be performed. During this second examination, a prolapse of hyperemic mucosa was observed where the polyp had previously been visible ( $\mathbf{F i g . 2}$ ). On closer inspection, the tip of the stalk had an area of minimal erosion surrounded by an area of surface healing. The patient denied any symptoms such as pain or hematochezia. We hypothesized that polyp amputation had occurred just after the diagnostic colonoscopy, with the prolapsed mucosa being the remnant of the stalk of the amputated polyp. Histopathologic evaluation of biopsy specimens from the prolapsed mucosal lesion and the surrounding mucosa, which was of normal appearance, revealed no abnormal findings.

At follow-up colonoscopy 8 months later, there was dimpling of the mucosa covering a near-normal surface ( $\bullet$ Fig. 3 ). The height of the remnant stalk had decreased compared with the previous examination. Histopathology again showed no abnormal findings.
Disappearance of a polypoid lesion in the gastrointestinal tract is a rare phenomenon. Its precise mechanism remains unknown [1 -5], but several possible mechanisms have been proposed. This unusual occurrence may follow twisting of the pedicle/stalk [1,2], ulcerogenic medications [3], or necrosis and cancerous infiltration of the polyp base [4]. External force caused by instrumentation such as the use of biopsy forceps [5] or band ligation is another possible cause of detachment. Pedunculated polyps in particular may be easily distorted and are subject to mechanical tension. Therefore, during the examination, the colonoscope may produce friction with the polyp, especially if it is large, and can cause torsion of the polyp.Ischemic necrosis and tearing of the polyp base or amputation can follow, as was believed to have occurred in the present case.

Disappearance of the polyp in our patient was not autoamputation. We believe that the most likely explanation for this phenomenon was colonic injury caused by the colonoscope. To our knowledge, amputation of a colonic polyp by a colonoscope has not previously been reported. The present case is the first to document the disappearance of a polyp following a diagnostic colonoscopy.

Endoscopy_UCTN_Code_CCL_1AD_2AB

Competing interests: None

\section{H. W. Lee, W. Moon, S. J. Park, M. I. Park, H. H. Kim, J. M. Choi}

Department of Internal Medicine, Kosin University College of Medicine, Busan, Korea

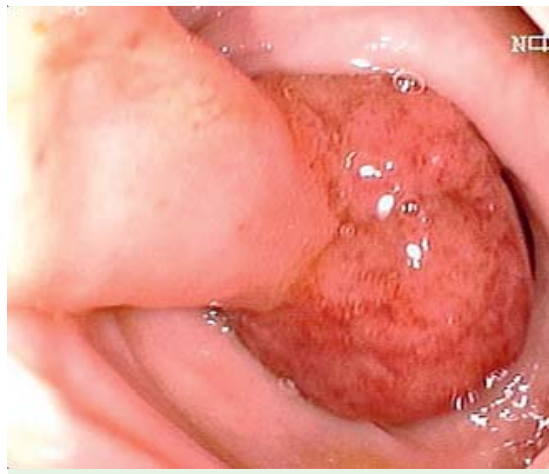

Fig. 1 Colonoscopy performed at another hospital in a 66-year-old man showing a large pedunculated polyp with an erythematous surface that was occupying almost the entire lumen in the mid-sigmoid colon.

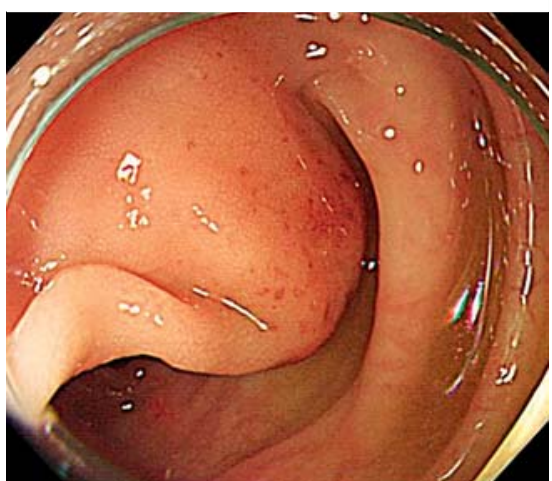

Fig.2 A second colonoscopy performed for polypectomy to be undertaken showing prolapse of hyperemic mucosa where the polyp had previously been visible.

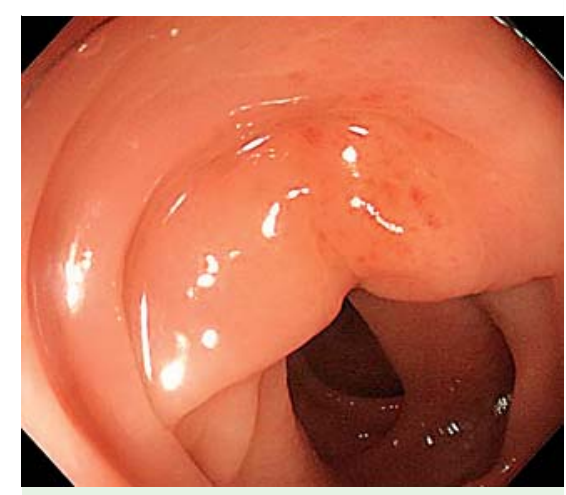

Fig. 3 Follow-up colonoscopy 8 months later showing dimpling of the mucosa covering a near-normal surface. The height of remnant stalk had decreased compared with the previous examination. 


\section{References}

1 Kaneki $T$, Kawashima A, Tsushima $K$ et al. Submucosal tumor-like elevated lesion after auto amputation of a colonic polyp. Gastrointest Endosc 2002; 55: $444-446$

2 Jeong HK, Cho SB, Seo TJ et al. Autoamputation of a giant colonic lipoma. Gut Liver 2011; 5: 380-382

3 Nakajima T, Kamano T, Watanabe $K$ et al. A gastric hyperplastic polyp observed endoscopically before and after autoamputation. Endoscopy 2003; 35: 1069-1071
4 Furusawa M, Koga M, Soejima K et al. A protruding type of advanced carcinoma of the stomach followed up as a polyp for four years, report of a case [in Japanese with English abstract]. Stomach Intest (Jpn) 1973; 8: 597-603

5 Tsukamoto $Y$, Nishitani $H$, Oshiumi $Y$ et al. Spontaneous disappearance of gastric polyps: report of four cases. AJR Am J Roentgenol 1977; 129: 893-897

\section{Bibliography}

DoI http://dx.doi.org/

10.1055/s-0032-1326493

Endoscopy 2013; 45: E169-E170

(c) Georg Thieme Verlag KG

Stuttgart · New York

ISSN 0013-726X

\section{Corresponding author}

\section{W. Moon, MD}

Department of Internal Medicine Kosin University College of Medicine 34 Amnam-dong, Seo-gu

Busan 602-702

Korea

Fax: +82-51-9905055

moonone70@hanmail.net 